\title{
Determinants and Current Level of Optimal Complementary Feeding Practices among Lactating Mothers of Children in Ambo Town, Oromia, Ethiopia
}

\author{
Abebe Haile $^{1^{*}}$ and Assefa Belay ${ }^{2}$ \\ College Development Studies, Centre for Food Security Studies, Addis Ababa University, Addis Ababa, Ethiopia \\ Correspondence should be addressed to Abebe Haile, abebe.haile@gmail.com ; abebe.haile@aau.edu.et
}

Received: February 26, 2020; Accepted: March 22, 2020; Published: March 29, 2020

\begin{abstract}
An appropriate diet is critical in the growth and development of children especially in the first two years of life. Poor complementary feeding of children aged 6 months - 23 months contributes to the characteristics negative growth trends and deaths observed in developing countries. Therefore, this study aimed to assess determinants and current level of optimal complementary feeding practices among mothers of children aged 6 months to 23 months in Ambo town, Oromia Region. The study used cross sectional study design and targeted 336 mothers with children 6 months - 23 months olds. Information from the respondents were collected using standard questionnaire. Data entry and analysis was done using SPSS version 21.0 windows statistical software. All (100\%) the children 6 months - 8 months old had received solid, semi-solid/soft foods. The minimum meal frequency was attained by $88.3 \%$ (95\% CI 84.3-91.4) whereas the minimum dietary diversity was attained by $17.9 \%$ (95\% CI 14.1-22.5). The minimum acceptable diet was attained by 15.4\% (95\% CI 11.9-19.8). Maternal knowledge on: importance of breastfeeding (87.3\%); age of introduction of complementary foods (85.4\%) and correct meal frequency for age (74.5\%) was high. On the contrary, knowledge on the importance of enriching complementary foods (34.5\%) was low. Mothers who knew the importance of a diverse diet were likely (chi-square test; $\mathrm{p}=0.001$ ) to feed their children on a diverse diet. On the other hand, mothers who knew the importance of enriching complementary foods were likely to feed their children on a minimum acceptable diet (chi-square test; $\mathrm{p}=0.007$ ) and maternal knowledge on enriching complementary foods $(\mathrm{OR}=3.41, \mathrm{p}=0.040)$ were significant predictors of consumption of Vitamin A rich foods, minimum meal frequency and minimum acceptable diet, respectively. Behaviour change and communication involving all the stakeholders in infant and young child feeding should be emphasized. Messages on appropriate feeding practices should include importance of dietary diversity.
\end{abstract}

\section{KEYWORDS}

Complementary feeding; Breastfeeding; Children age 6-23 months; Determinant

Citation: Abebe Haile, Determinants and Current Level of Optimal Complementary Feeding Practices among Lactating Mothers of Children in Ambo Town, Oromia, Ethiopia. Food Proc Nutr Sci 1(1): 31-50.

(C)2020 The Authors. Published by TRIDHA Scholars. 


\section{INTRODUCTION}

Malnutrition remains one of the most common causes of morbidity and mortality among children throughout the world. It has been responsible, directly or indirectly, for $60 \%$ of the 10.9 million deaths annually among children under five and two-thirds of these deaths, which are often associated with inappropriate feeding practices [1]. Over one third of under-five mortality is caused by malnutrition related to inappropriate complementary feeding. Initiate safe and nutritionally adequate complementary foods at 6 months is crucial to achieve optimal growth, development and health [2]. An appropriate diet is a critical component for proper growth and development of children [3]. The first two years of life are a critical window for ensuring optimal child growth and development [4]. Nutritional deficiencies during this period can lead to impaired cognitive development, compromised educational achievement and low economic productivity which become difficult to reverse later in life [4]. Improving infant and young child feeding (IYCF) practices in children 0 months

- 23 months of age is therefore critical to improved nutrition, health and development [1]. Scientific evidence indicates that inappropriate feeding practices can have profound consequences for the growth, development and survival of infants and children [5].

Various inappropriate complementary feeding practices such as; untimely introduction of complementary food, improper feeding frequency and low dietary diversity of complementary foods have been shown to have numerous negative effects on children's health. Appropriate complementary feeding entails; introduction of complementary foods at 6 months with continued breastfeeding up to at least 2 years and beyond, correct feeding frequency for age and consumption of a diverse diet [6]. There is strong evidence that the promotion of appropriate complementary feeding practices reduces the incidence of stunting and leads to better health and growth outcome. Therefore, as an effective intervention strategy for malnutrition, WHO and UNICEF recommended introduction of adequate complementary foods at 6 months with continued breastfeeding for 2 years of age or beyond [7,8]. In sub-Saharan African Regions, suboptimal infant feeding practices, poor quality of complementary foods, micronutrient deficiencies and frequent infections have mainly contributed to the high mortality among infants and young children. Similarly, malnutrition is a significant health problem for infants and young children in Ethiopia. In Ethiopia, age appropriate infant and young child feeding practice is alarmingly low; only 7 percent of children age 6 months - 23 months have met the criteria for a minimum acceptable diet [9]. Another study conducted using composite indicators, Ethiopia Demographic Health Survey (EDHS) [10] and study conducted in Northern Ethiopia showed the level of appropriate complementary feeding to be too low, $7 \%$ and $15 \%$ respectively. To improve complementary feeding practice through this essential time of growth and development of the child, assessment of complementary feeding practices and its factors are vital.

The most recent estimates of the global burden of malnutrition in children younger than five years of age show that 178 million are stunted, 112 million are underweight and 55 million are wasted. Together they account for $21 \%$ of all under-5 deaths [11], with over twothirds of these deaths occurring during the first year of life. Malnutrition is also a direct cause of mortality, and a major disabler preventing children who survive to reach their full developmental potential [12]. In the causal matrix of undernutrition [13], an important underlying determinant of child under-nutrition is the care provided to the child. The key care practices that could impact on child nutritional status include breast feeding and complementary feeding. Exclusive Breast Feeding (EBF) for the first 6 months of life followed by optimal complementary feeding are critical public health measures in ensuring good nutritional status and reducing mortality significantly [14]. Appropriate complementary feeding which comprise introduction of 
complementary foods at 6 months with continued breastfeeding to at least 2 years, correct feeding frequency for age and consumption of a diverse diet can prevent $6 \%$ of child deaths per year [15]. There is, therefore, sufficient reason to both prevent and appropriately manage malnutrition in early childhood through appropriate child feeding, if both the short-and long-term consequences are to be avoided [16]. Complementary feeding refers to gradual dietary transition characterized by introduction of solid and semisolid foods to an infant's diet when breast milk alone becomes insufficient in meeting the nutritional needs of the infant. The recommended age range for complementary feeding is generally taken to be 6 months to 24 months even though breastfeeding may continue beyond two years [7]. The nutritional inadequacy of the complementary diet, both in quality and quantity, and the undermining effects of infections on the nutritional status of the child remain major problems affecting infants and young children in the world today [17]. Guidelines on complementary feeding outlined in the Ethiopian National Policy on Infant and Young Child Feeding, as adopted from the WHO recommendations, are comprehensive. They include the following: Commencing complementary feeding at six months with small amounts of food increased gradually as the child gets older. Food variety and consistency should also be gradually increased. Another recommendation is frequent, on-demand breastfeeding until two years of age or beyond and the use of responsive feeding which directs that infants are fed directly and older children assisted with eating. It is also recommended that infants and young children be slowly and patiently fed, thus encourage eating without use of force [9].

Poor complementary feeding practices have been widely documented in Ethiopia despite the government and other stakeholders implementing a number of strategies aimed at improving IYCF practices [9]. In line with this, a significant proportion of infants and young children in Ethiopia are exposed to the hard consequences of poor complementary feeding practices and the situation is likely to be worse among the urban poor [18]. Despite all these, the prevalence of appropriate complementary feeding practices in different part of Ethiopia are lower than international recommendation. In 2016, nearly 4 in 10 $(38 \%)$ of children under five in Ethiopia are stunted, or too short for their age [10]. Inappropriate complementary feeding practices such as untimely introduction of complementary foods, improper feeding frequency and low dietary diversity of complementary foods have been widely shown to increase the risk of underweight and stunting especially among the urban poor [19]. Study done in Oromia region showed that more than half of the caregivers or mother did not timely initiated complementary feeding. The main reason for too early initiation of complementary feeding was lack of knowledge and perceive inadequate breast milk production. There was limited scientific data on complementary feeding practices and its relation to the nutritional status of children 6 months - 23 months old in urban poor in Ethiopia. These evidences strongly call for the need to improvement of complementary feeding practices but there is limited scientific data on complementary feeding practices that captured the multidimensionality of feeding practices including dietary diversity, frequency, and acceptable diet and associated factors in the region, especially, in the study area. To improve complementary feeding in low-resource settings during this critical period of growth and development, assessment of optimal complementary feeding practices is essential. Therefore, this study aimed to assess level and determinants of optimal complementary feeding practices among mothers of children aged 6 months to 23 months in Ambo town, Oromia region.

\section{Conceptual framework for the study}

The variables under study have been represented diagrammatically to show the relationship between them by illustrating the influence of the independent variables on the dependent variable in order to give coherence to this report (figure 1). The study used a conceptual framework adapted by the researcher from the determinants factors of 
complementary feeding practices in different states of the world's children report. Proximate determinants were a result of inter-related underlying factors, encompassing: poor complementary feeding practices; inadequate care givers knowledge and inappropriate complementary feeding practices which consist of untimely introduction, improper feeding frequency and low dietary diversity of complementary foods result to inadequate dietary intake of the infants and children.
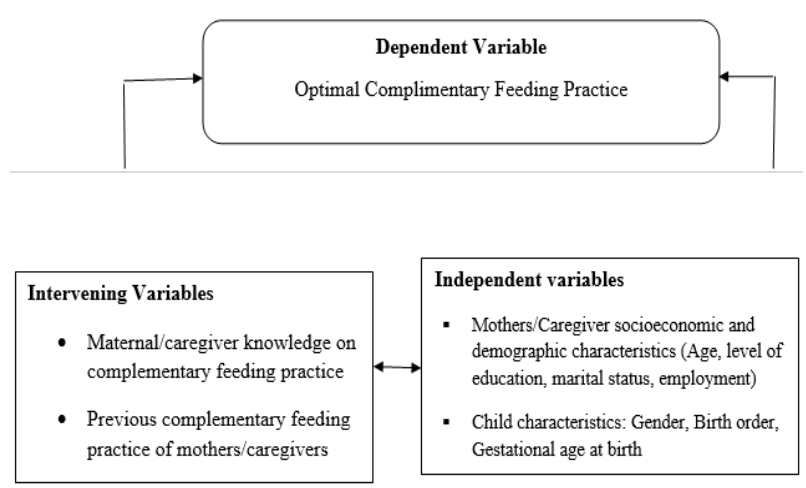

Figure 1: Adopted and modified conceptual framework.

\section{RESEARCH METHOD AND DESCRIPTION OF STUDY AREA}

\section{Description of study area}

The study was carried out in Ambo town, which is one of the 22 woredas of West Shoa administrative zone of Oromia, Regional State. It is located $114 \mathrm{~km}$ west of Addis Ababa. As indicated in figure 2 astronomically it is located at about 9045'28" - 9048'16" North latitude and 38021'55" - 38023'45" East longitude and an elevation of 2101 meters above sea level.

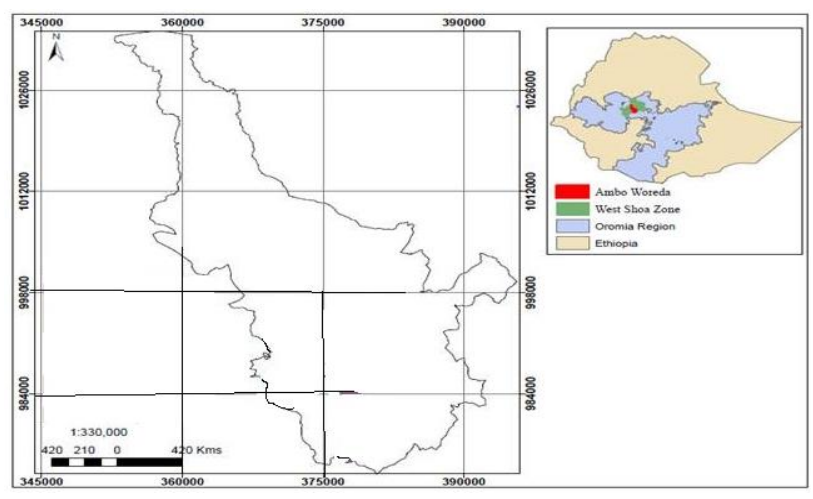

Figure 2: Location of Ambo town in its national and regional settings. (Source: http://www.maps.world.com).
According to information obtained from Ambo Town Health office, there are estimated projected total populations of 83,053 in Ambo town of which about 41,061 are women and the rest are males based on 2009 Ethiopian Fiscal Year (EFY) of Central Statistics Agency estimation. There are six kebeles in the town namely $(01),(02),(03)$, Senkele (04), Kisose (05) and Awaro (06) and with total population of 40018, 16725, 9163, 7968, 5976 and 3203, respectively. There are also 17,303 households and Children aged 6 months - 23 months of age in the town constituting $5.21 \%$ (4319) of the population $(2083,870$, $476,414,310$ and 166, respectively). In the town, there is one referral hospital, one general hospital, two health centers, three health posts, twenty-eight private clinics and one non-governmental organization working on maternal and child health. According to the Ambo Town health office 2017 annual report, malnutrition is one among the ten top diseases.

\section{Study design and period}

Community-based cross-sectional study was employed to collect relevant and sufficient information within short period of time. The study design was used quantitative and qualitative research approach to assess determinants and current level of optimal complementary feeding practice among mothers of infants and young children aged 6 months to 23 months in Ambo town; from February 15 to March 15, 2019.

\section{Population}

The source populations were mothers having children in age group of 6 months to 23 months residing in the study area. Study population was randomly selected mothers with children aged 6 months - 23 months and resided in the study area for more than 6 months.

\section{Inclusion and exclusion criteria}

Inclusion criteria was the mothers/caregivers of children and infants 6 months - 23 months old who were residents of Ambo town for the past 6 months and are willing to 
participate in the study. Whereas, the mother who declines to participate in the study and mother of very sick infants and young children, those with known anomalies or those requiring emergency care at the time of the study was not include in the study. The status of the above conditions was determined based on mother/caregiver self-reports, observation and records on the child health card.

\section{Sample size determination}

To determine the children to be included in the study different methods were employed in order to get representative of sample size. Therefore, cochran (1963:75) formula to yield the required sample for proportions was used as cited by Israel (1992). Thus,

$$
\frac{\mathrm{n}=(\mathrm{Z} \alpha / 2)^{2} \mathrm{pq}}{\mathrm{d}^{2}}
$$

Where: $\mathrm{n}=$ is the desired sample size; $\mathrm{Z}=\mathrm{Z}$-Score is the standard normal deviation at a confidence level set at $95 \%$ which is 1.96; $\mathrm{p}=$ estimated proportion of an attribute that is present in the population; $\mathrm{q}=\mathrm{p}-1 ; \mathrm{d}=$ desired level of precision.

To estimate the sample size the expected prevalence of complementary feeding practices for the study area is not known. Therefore, assume $\mathrm{p}=0.50$ (maximum expected prevalence). Accordingly, the desired level of precision 5\% with $95 \%$ level of confidence the $\mathrm{Z}$ value equals 1.96 the sample size/n $=384$. The total population of the town is 1552; need to adjust using finite population correlation factors. Finite population correction factor applied when the sample represents a significant (e.g. over 5\%) proportion of the population. The formula should be:

$$
n_{a}=\frac{n}{1+\frac{n-1}{N}}
$$

Where: $\mathrm{n}_{\alpha}=$ required sample size; $\mathrm{N}=$ total population of the mothers of children age 6-23 months in the study area; $\mathrm{n}=$ sample size estimated based on the assumption of $\mathrm{p}=$ 0.50; Thus, the final sample size was 339 and with nonresponse rate of $10 \%$.

\section{Sampling techniques}

A multi stage stratified sampling was used, considering old and new as strata, two Kebeles from the old and two Kebeles from the new, then 4 Kebeles was randomly selected from both new and old using simple random sampling (SRS) method. The projected total population size in the four selected kebeles is 60,352 of which 3,139 is children 6 months - 23 months of age. List of households with children 6 months to 23 months in the selected kebeles was obtained from health extension office before data collection. By proportional to size (PPS) sampling technique, Study participants was allocated to select four Kebeles 339 households with eligible children 6 months to 23 months was selected using systematic random sampling technique from households with children 6 months to 23 months in this four Kebeles. Members of the focus group discussions (FGDs) were purposively and conveniently selected to take part with the help of village elders and health extension workers (HEWs). This ensured that participants who were easily accessible and have adequate information regarding infant feeding in the study area were selected. To enhance homogeneity, each FGD participants were made up of 6 mothers to 12 mothers with children below 2 years who was not part of the main sample. The FGDs were held in all the four Kebeles after quantitative data had been collected. In short, the schematic procedure of sampling techniques of this study is shown in the following (Figure 3).

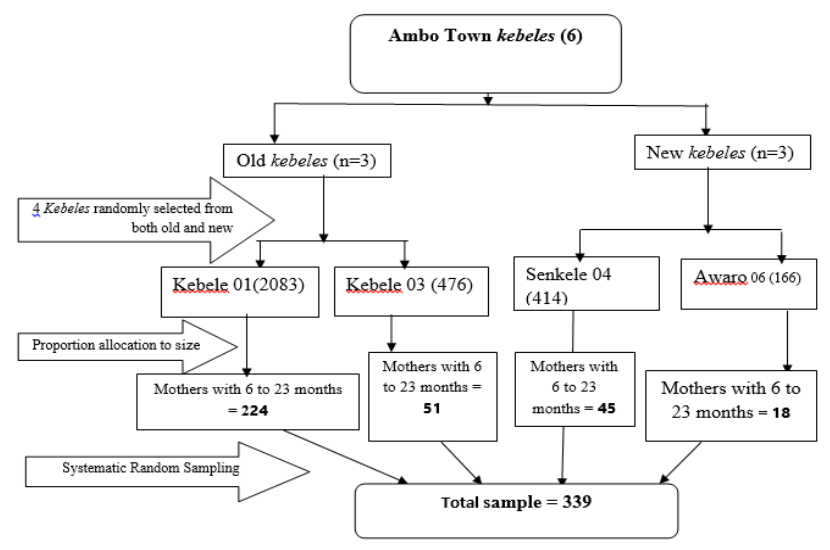

Figure 3: Schematic procedures of sampling techniques used for the selection of participants. 


\section{Data collection instrument and procedure}

As indicated earlier the research design that was employed in this study is partially mixed quantitative and qualitative method. Therefore, in addition to socio-demographic and economic characteristics, quantitative and qualitative data was generated by using survey questionnaire, focus group discussions and key informant interviews. The pre-testing was conducted to establish accuracy of questions and clarity and to determine the length of interviews. During pre-testing an effort was made to check for consistency in the interpretation of questions and to identify ambiguous items. After review of the instruments all suggested revisions was made before being administered in the actual study.

\section{Questionnaire survey}

This was vital data acquisition technique in this study. A semi-structured questionnaire consisting of closed and open-ended questions was used to elicit responses from the study participants. The socio-demographic and economic characteristics of respondents, Infants characteristics, maternal knowledge and previous complementary feeding practices and intakes of complementary foods using 24hours recall adopted from the WHO infants and Young child feeding indicator questionnaire [20]. In addition, some modification of the questioner was done based on the objectives and local situation of the area. The questionnaire captured data on each of the objectives of the study and was coded to facilitate data entry analysis. Data was collected by self-administered questionnaire to the mother/caregiver in face-to-face interviews during a one-time visit to the household. The questionnaire was first prepared in English and later translated into Afan Oromo and back translated into English to check for its conceptual equivalence. Mothers were asked specific questions to elicit information on socio-demographic and economic characteristics, regarding practices of complementary feeding of the child, mothers were requested to respond the age at which complementary feeding started, types of complementary feeding given for their child, frequency of complementary feeding in per/day and time of breastfeeding stopped from the child. The Diet Diversity Score (DDS) and a 24-hours recall method was conducted with mothers regarding their child's intake. Mothers/caregivers was requested to list all the foods consumed by the child both at home and other places. The consent of each respondent were also be sought and secured prior to the commencement of the actual survey processes.

\section{Key informant interview}

Key informant interviews were carried out with the intention of capturing more information on optimal complementary feeding. The key informants were comprised of the elderly, health extension workers, health administration officials and managers of kebeles. Each interview was carried out by the researcher with the aim of making further investigations based on the information received from the respondents.

\section{Focus group discussion (FGD)}

Four focus group discussions were carried out with representatives from different economic status (well-off and indigent), religion, gender, age group and communitybased organizations. This technique were used to extract information in a participatory manner so that the perceptions and views of the community were captured and interpreted. Suitable conditions were set for the discussants so that they would be able to describe the issues under investigation precisely in their own language, Afan Oromo. The participants were respectfully requested for their time. Topics related to infants and children feedings, determinants of complementary feedings, knowledge and perceptions on the adequacy of complementary feeding in community was addressed. Each FGD should a minimum of 6 and a maximum of 12 mothers/caregivers with children below 2 years. Members of the FGDs were recruited by the supervisors with the help of community leaders, Health Extension Workers and village elders. The supervisors moderate the discussions while one of his assistants took 
notes and the discussions were tape recorded and nonverbal communication documented.

\section{Data quality assurance}

To ensure the quality of research data, data collectors, and supervisors were take one-day training on the objective, methodology, sampling technique, ethical issues, and data collection instrument and data collection procedure. In addition, the data collection instruments were pretested on $5 \%$ sample size of Ambo town. The principal investigator was checked the quality of data collection process.

\section{Study variables}

The dependent variables for the study were optimal complementary feeding practices. While the independent variables were socio-demographic characteristics of mothers or guardians and infants (Age, sex, residence, marital status, occupational status, educational status, income, religion, ethnicity, parity, household size); maternal knowledge and perception on complimentary feeding practices.

\section{Data processing and Analysis}

All the collected data was checked for completeness and internal consistency by cross checking, then was coded, and double enters for analysis; the data was exported to Statistical package for social science (SPSS) version 21.0 software. Descriptive statistics was used to describe sociodemographic and economic characteristics, health related characteristics, and complementary feeding indicators of infants. To test for the association between two variables such as material or infant characteristics and infant and children feeding practices, Chi-square was used to determine significance of association. Systematic Random Sampling and bivariate analysis was used to prevent selection bias and variable confounder effects, respectively. Logistic regression was used to identify predictors of complementary feeding practices. A P-value of $<0.05$ was used as the criterion for statistical significance. Data from FGDs was transcribed, coded and common themes established. Selected responses from FGDs were directly quoted to exemplify common perceptions among the respondents.

\section{Ethical consideration}

Ethical approval was taken from Addis Ababa university school of graduate studies ethical board, obtained a letter of authority to conduct the study. Official letter was taken to Ambo town administrative office for commencing the study, the data collection was beginning after permission, and cooperation letter was written to each selected Kebeles authorities on which the study was carried out. At the house hold level, informed signed or thumb print consent was sought from the respondents. The study, purpose, procedure and duration, possible risks and benefits of the study were clearly explained for the participants using local language. Respondents were guaranteed confidentiality and informed that the information provided was only be used for research purposes. Confidentiality was assured by not including respondent names on the questionnaires but only identity numbers.

\section{RESULT AND DISCUSSION}

The data was collected using close ended questionnaire. The questionnaires were self-administered to the respondents. Out of 339 questionnaires that were issued 336 questionnaires were returned. This represents about a response rate of $99.1 \%$, which was significant to give reliable findings for this study (Table 1). A low response rate could have a potentially biasing effect on the study results. However, $70 \%$ and above response rate is acceptable for any study.

\begin{tabular}{|l|r|r|}
\hline & Frequency & Percentage \\
\hline Non Respondent & 3 & $0.90 \%$ \\
\hline Actual Respondent & 336 & $99.10 \%$ \\
\hline Target population & 339 & $100 \%$ \\
\hline
\end{tabular}

Table 1: Response rate in Ambo town, Oromia region, Ethiopia, 2018/9. 


\section{Socio-demographic profiles of the households}

Three hundred and thirty six (336) mothers and caregivers representing the same number of households were interviewed during the study. The median age of the mothers was 24 years with the youngest and oldest mothers being 16 years and 54 years old respectively. Majority of the study participants $(87.5 \%)$ were Oromo by ethnicity and more than half $(55.1 \%)$ protestant religion followers followed by orthodox religion (41.4\%). Majority (74.5\%) of the mothers were married, $16.1 \%$ single, $6.5 \%$ widowed while $2.8 \%$ were separated. Over half $(59.6 \%)$ of the mothers had primary school education while $29.5 \%$ had secondary education. The average size of a household was 4.6(range 1-9) people (Table 2).

\begin{tabular}{|c|c|c|}
\hline Socio-demographic characteriatics & $\mathrm{N}=336$ & \\
\hline & $\mathrm{N}$ & $\%$ \\
\hline \multicolumn{3}{|l|}{ Matemal/caregiver age (years): } \\
\hline Median (range) & $24(16-54)$ & \\
\hline$<25$ years & 189 & 56.2 \\
\hline $25-34$ years & 119 & 35.4 \\
\hline 35 years and above & 28 & 8.4 \\
\hline Ethnicity: & 294 & 88 \\
\hline \multicolumn{3}{|l|}{ Oromo } \\
\hline Ambara & 31 & 9 \\
\hline Guraghe & 10 & 3 \\
\hline Others* & 1 & 0 \\
\hline Religion: & 139 & 41 \\
\hline \multicolumn{3}{|l|}{ Orthodox } \\
\hline Protestant & 185 & 55 \\
\hline Muslim & 10 & 3 \\
\hline Wlakefata & 2 & 1 \\
\hline \multicolumn{3}{|l|}{ Marital status: } \\
\hline Single & 54 & 16.1 \\
\hline Married & 250 & 74.5 \\
\hline Separated & 10 & 2.8 \\
\hline Widowed & 22 & 6.5 \\
\hline \multicolumn{3}{|l|}{ Education: } \\
\hline No formal education & 19 & 5.6 \\
\hline Primary school level & 200 & 59.6 \\
\hline Secondary school level & 99 & 29.5 \\
\hline Tertiary level & 18 & 5.3 \\
\hline \multicolumn{3}{|l|}{ Parity (number of Children): } \\
\hline Median (range) & $2(1-7)$ & \\
\hline \multicolumn{3}{|l|}{ Household size: } \\
\hline Mean (range) & $4.63(1-9)$ & \\
\hline
\end{tabular}

Table 2: Socio-demographic characteristics of the study population in Ambo Town, Oromia region, Ethiopia, 2018/9.

\section{Socio-economic profiles of the households}

Over three quarters of the families $(77.0 \%)$ depended on casual labour as their main source of income followed by small-scale business $(16.1 \%)$ and lastly formal employment $(6.8 \%)$. Nearly all $(94.1 \%)$ the households obtained food through purchasing from the market. Nearly half $(46.3 \%)$ of the households estimated to allocate medium percentage $(30 \%-65 \%)$ of their income to food while $(39.4 \%)$ allocated the largest percentage $(>65 \%)$ of their income to food and only (14.3\%) allocated the smallest percentage $(<30 \%)$ of their income to food (Table 3).

\begin{tabular}{|c|c|c|}
\hline Socio-economic characteristics & \multicolumn{2}{|c|}{$\mathbf{N}=\mathbf{3 3 6}$} \\
\cline { 2 - 3 } & $\mathbf{N}$ & $\mathbf{\%}$ \\
\hline Main source of family income: & & \\
\hline Formal employment & 23 & 6.8 \\
\hline Casual labour & 259 & 77 \\
\hline Small scale business & 54 & 16.1 \\
\hline Estimated \% household of income allocated to food: & & \\
\hline Largest percentage (>65\%) & & \\
\hline Medium percentage (30\%-65\%) & 132 & 39.4 \\
\hline Smallest percentage (<30\%) & 156 & 46.3 \\
\hline How food is obtained: & 48 & 14.3 \\
\hline Farming & & \\
\hline Purchase & 14 & 4 \\
\hline Food aid/donation & 316 & 94.1 \\
\hline Others & 4 & 1.2 \\
\hline Provider* of food in a household: & 2 & 0.6 \\
\hline Father/Husband & & \\
\hline Mother & 257 & 76.4 \\
\hline Grand parents & 70 & 20.8 \\
\hline Relatives & 4 & 1.2 \\
\hline & 5 & 1.6 \\
\hline
\end{tabular}

Table 3: Socio-economic characteristics of the of the study population in Ambo town, Oromia region, Ethiopia, 2018/9.

Feeding practices among children 6 months - 23 months old in Ambo town

Infant feeding practices were measured by a set of simple, valid and reliable indicators developed by WHO over a period of 5 years. The indicators focus on selected food related aspects of child feeding associated with both breastfeeding and complementary feeding, amenable to population level measurement. Even though the study focused on complementary feeding, it was worth to give a snapshot of some breastfeeding practices.

\section{Breastfeeding practices}

Nearly all the children (92.0\%) had ever been breastfed with slightly over two-thirds $(68.8 \%)$ having been initiated to breastfeeding timely (within 1 hour of birth). Whereas, half $(50.0 \%)$ of those who never breastfed was due to the fact that their mothers had no breast milk others $(42.3 \%)$ chose not to breastfeed their children (Table 4). 


\begin{tabular}{|c|c|c|c|}
\hline \multirow[t]{2}{*}{ Breastfeeding practices } & \multicolumn{3}{|c|}{$\mathrm{N}=336$} \\
\hline & $\mathbf{N}$ & $\%$ & $95 \% \mathrm{CI}$ \\
\hline Ever breastfed $\mathrm{N}=336$ & 309 & 92 & \\
\hline Timely initiation of breastfeeding $\mathrm{N}=336$ & 231 & 68.8 & \\
\hline \multicolumn{4}{|l|}{ Reasons for not breastfeeding: $\mathrm{N}=26$} \\
\hline Did not have milk & 13 & 50 & \\
\hline Did not want to breast feed & 11 & 42.3 & \\
\hline Other reasons & 2 & 7.7 & \\
\hline Children 6-23 months still breastfeeding $N=298$ & 265 & 88.9 & $84.8-94.0$ \\
\hline Continued breastfeeding at 1 year $\mathrm{N}=79$ (children $12-15$ months old) & 69 & 87.3 & $78.1-93.2$ \\
\hline Continued breastfeeding at 2 years $\mathrm{N}=49$ (children $20-23$ months old) & 32 & 65.3 & $51.3-77.1$ \\
\hline Bottle feeding $\mathrm{N}=336$ & & 29.6 & \\
\hline \multicolumn{2}{|l|}{ Age of cessation of breastfeeding (months) $\mathrm{N}=33$} & & \\
\hline Mean, (SD), range & & & \\
\hline Apr-18 & & & \\
\hline
\end{tabular}

Table 4: Breastfeeding practices in Ambo town, Oromia region, Ethiopia, 2018/9.

Majority (87.3\%, 95\% CI; 78.1-93.2) of the children 12 months - 15 months old were still breastfeeding compared to their 20 months - 23 months old counterpart who rated slightly lower at $(65.3 \%, 95 \%$ CI $78.1-93.2)$. During the FGDs, mothers reported that it was challenging to breastfeed up to 2 years and beyond due to lack of breast milk and occupations, which kept them away from, home most of the time. Slightly above a 38 quarter of the children (29.6\%) received food or drink from a bottle with a nipple/teat in the previous day (Table 4).

\section{Complementary feeding practices}

Introduction of solid, semi-solid or soft foods

All the children 6 months - 8 months (100\%) had received solid, semi-solid or soft foods the previous day. During the FGDs, mothers reported to have introduced other foods apart from breast milk as early as when their babies were 2 months old.

\section{Dietary diversity of complementary feeding diet}

Dietary diversity was determined based on a 24-hours recall. The mothers were requested to state what their children consumed the previous day. Dietary diversity was then computed based on 7 food groups as recommended by [1] which comprise of: grains, roots and tubers; legumes and nuts; dairy products; flesh foods (meat, fish, poultry and organ meats); eggs; vitamin-A rich fruits and vegetables; other fruits and vegetables. Consumption of any amount of food from each food group was sufficient to count except if a food item was only used as a condiment. Nearly all the children (94.4\%) consumed foods made from grains, roots and tubers. Over half of the children consumed grains, roots and tubers. Vitamin-A rich fruits and vegetables were consumed by $47.8 \%$, dairy products by $32.1 \%$, other fruits and vegetables by $31.2 \%$ and finally legumes and nuts by $18.2 \%$ of the children aged 6 months - 23 months old. Consumption of animal origin foods was low, barely $4.6 \%$ of the children consumed eggs while consumption of dairy products and flesh foods was at $32.1 \%$ and $13.0 \%$, respectively (Figure 4 ).

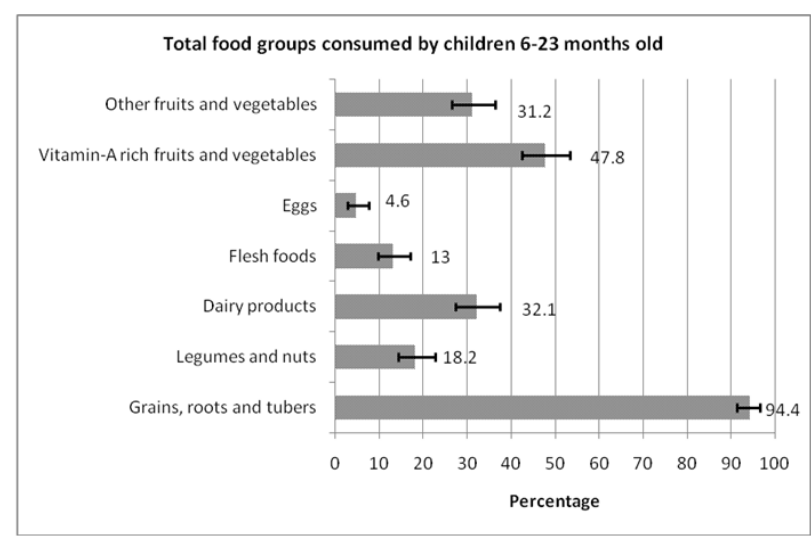

Figure 4: Types of food given to children aged 6 months 23 months in Ambo town, Oromia region Ethiopia, 2019.

The mean dietary diversity score for children aged 6 months - 23 months old was 2.4, ( $\pm 1.3,95 \%$ CI 2.3-2.6) and the scores ranged from 1 to 7 . Slightly over one-tenth (13.6\%) of the children aged 6 months - 23 months old consumed iron-rich and iron fortified foods. The percentage of those who consumed iron-rich foods was more or less the same in the other age categories with $10.5 \%$ in 6 months - 11 months, $15.7 \%$ in 12 months - 17 months and $16.5 \%$ in 18 months - 23 months old (Table 5). To determine minimum dietary diversity, a cut-off of at least 4 out of the above listed 7 groups was selected because it is associated with better quality diets for both breastfed and non-breastfed children [21]. Consumption of foods from at least 4 food groups on the previous day would mean that the children have a high likelihood of consuming at 
least one animal-source food and at least one fruit or vegetable that day, in addition to a staple food (grain, root or tuber) [12].

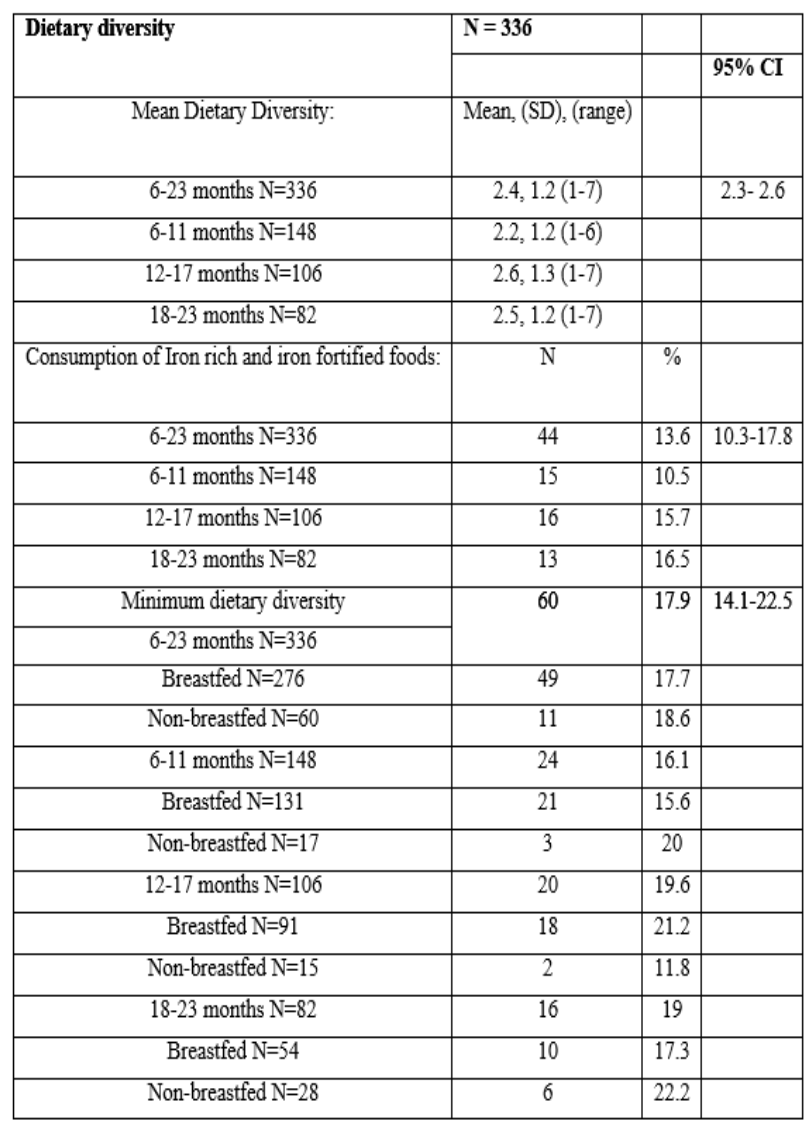

Table 5: Foods consumed and dietary diversity in Ambo town, Oromia region, Ethiopia, 2018/9.

Less than a two-tenths $(17.9 \%$, 95\% CI 14.1-22.5) of children 6 months - 23 months old received foods from 4 or more food groups in the previous 24 hours. In the same age category, $17.7 \%$ and $18.6 \%$ of breast fed and nonbreastfed children attained minimum dietary diversity respectively. Minimum dietary diversity was highest (19.6\%) in 12 months - 17 months old category (21.2\% and $11.8 \%$ for breast fed and non-breastfed children respectively). This was followed by 18 months -23 months old category (19.0\%) (17.3\% and $22.2 \%$ for breast fed and non-breastfed children respectively). The last was the 6 months - 11 months old category (16.1\%) (15.6\% and $20.0 \%$ for breast fed and non-breastfed children respectively) (Table 5).

\section{Meal frequency}

The mean meal frequency for all the aged children 6 months - 23 months old was $3.45( \pm 1.14)$ (95\% CI 3.3-3.6).

The number of meals consumed by the children in all the age categories ranged from 1 to 6 . The minimum meal frequency was achieved by most $(88.3 \%$; $95 \%$ CI $84.3-$ 91.4) of the children 6-23 months old with the same trend in the 6-11 months (86.7\%), 12 months - 17 months (92.2\%) and 18 months - 23 months (86.1\%) old age categories. The percentage of breastfed and non-breastfed children who attained minimum meal frequency in the different age categories was $88.3 \%$ and $88.1 \%$ in 6 months - 23 months old; $87.5 \%$ and $80.0 \%$ in 6 months - 11 months old; $91.8 \%$ and $94.1 \%$ in 12 months - 17 months old and $84.6 \%$ and $88.9 \%$ in 18 months - 24 months old respectively. During the FGDs mothers reported that children 6 months - 23 months old should consume at least 3 meals in a day (Table 6).

\begin{tabular}{|c|c|c|c|}
\hline Meal frequency & Mean, (range), (SD) & & 95\% CI \\
\hline Meal frequency: & & & \\
\hline Children 6-23 months old N 336 & $3.6,(1-6), 1.1$ & & $3.3-3.6$ \\
\hline 6-11 months (N=148) & $3.3,(1-6), 1.2$ & & \\
\hline $12-17$ months (N=106) & $3.6,(1-6), 1.0$ & & \\
\hline $18-23$ months (N=48) & $3.4,(1-6), 1.2$ & & \\
\hline Minimum meal frequency: & $\mathrm{N}$ & $\%$ & \\
\hline 6-23 months N=336 & 297 & 88.3 & $84.3-91.4$ \\
\hline Breastfed N=276 & 244 & 88.3 & \\
\hline Non-breastfed N=60 & 53 & 88.1 & \\
\hline 6-11 months N=148 & 128 & 86.7 & \\
\hline Breastfed N=131 & 115 & 87.5 & \\
\hline Non-breastfed N=17 & 13 & 80 & \\
\hline 12-17 months N=106 & 98 & 92.2 & \\
\hline Breastfed N=91 & 84 & 91.8 & \\
\hline Non-breastfed N=15 & 14 & 94.1 & \\
\hline 18-23 months N=82 & 71 & 86.1 & \\
\hline Breastfed N=54 & 46 & 84.6 & \\
\hline Non-breastfed N=28 & 25 & 88.9 & \\
\hline
\end{tabular}

Table 6: Meal frequency in Ambo town, Oromia region, Ethiopia, 2018/9.

Maternal/caregivers' knowledge on complementary feeding practices

In order to determine maternal knowledge on complementary feeding practices, mothers/caregivers were asked questions with regards to: initiation of semi-solid, 
solid or soft foods; dietary diversity and frequency of complementary foods; suitable preparation of complementary food and importance of breast feeding. Maternal nutrition knowledge was determined based on nutrition knowledge scores. Scores were coded as 1 for a correct response and 0 for an incorrect response, resulting in a total possible score of 12 . The overall nutrition knowledge score for each respondent was determined by the number of correct responses. Respondents with higher scores reflected higher nutrition knowledge on complementary feeding than those with lower scores.

\section{Knowledge on breastfeeding practices}

Majority of the mothers $(87.3 \%)$ interviewed knew that breastfeeding ensures proper growth and development and protects the baby from illness compared to the $69.9 \%$ who knew that breast milk alone can sustain the baby for the first 6 months of life (Table 7).

\begin{tabular}{|c|c|c|}
\hline Knowledge on breastfeeding & N & $\%$ \\
\hline Breastfeeding ensures proper growth and development and protects the baby from illness & 293 & 87.3 \\
\hline Breast milk alone can sustain the baby for the first 6 months & 235 & 69.9 \\
\hline Baby should be breast fed on demand & 232 & 69.1 \\
\hline Breastfeeding should continue for at least 2 years and beyond & 201 & 59.9 \\
\hline
\end{tabular}

Table 7: Maternal knowledge on breastfeeding in Ambo town, Oromia region, Ethiopia, 2018/9, $(\mathrm{N}=336)$.

\section{Knowledge on complementary feeding practices}

In the current study, most of the mothers $(85.4 \%)$ knew that semi-solid, solid and soft foods should be introduced at 6 months. Slightly less than three quarters of the mothers $(70.5 \%)$ did not know the risks of starting complementary feeding late. Malnutrition was the main consequence pointed out by mothers who knew the risk of introducing complementary feeding too late (Table 8 ). With regards to dietary diversity, mothers who stated that a child should consume a diverse diet were $65.8 \%$. Almost about $74.5 \%$ of the mothers were aware that a 6 months - 23 months old child should consume 2 meals or more in a day in addition to breast milk. Mothers' knowledge with regards to the importance of animal foods in complementary diet was low with only $18 \%$ of them pointing out that animal source foods are rich in nutrients and should form part of the complementary diet (Table 8). Slightly more than a third $(34.5 \%)$ of the respondents stated that enriching complementary food (through adding other foods like milk, fruit juices and fat to the main dish) makes it more nutrient dense/diverse and adequate to meet the dietary needs of the children especially as they grow. Just one-tenth $(7.5 \%)$ of mothers knew that child's porridge should be made of one type of flour. In addition to that mothers reported using mixed flour to prepare children's porridge during the FGDs (Table 8).

\begin{tabular}{|c|c|c|}
\hline Knowledge on complementary feeding practices & N & $\%$ \\
\hline Introduction of semi-solid, solid and soft foods: & & \\
\hline Complementary foods should be introduced at 6 months & 287 & 85.4 \\
\hline Starting complementary feeding after 6 months may cause malnutrition N $=336$ & 99 & 29.5 \\
\hline Dietary diversity and frequency of feeding: & 221 & 65.8 \\
\hline A child should consume a diverse diet & & \\
\hline A child should consume more than 2 meals per day & 250 & 74.5 \\
\hline Animal source foods are nutrient rich & 61 & 18 \\
\hline
\end{tabular}

Table 8: Maternal knowledge on complementary feeding practices in Ambo town, Oromia, Ethiopia, 2018/9, ( $\mathrm{N}=$ 336).

\section{Maternal/caregivers' knowledge score on complementary feeding}

The overall mean maternal knowledge score on complementary feeding was $6.8,( \pm 1.7)$ out of a total score of 12 and scores ranging from 3 to 10 (Table 9).

\begin{tabular}{|l|c|c|}
\hline Overall maternal/caregivers' knowledge score on complementary feeding & \multicolumn{2}{|c|}{$N=336$} \\
\hline Mean overall score: & & \\
\hline Mean, (SD), (range) & $6.47,(1.6),(3-10)$ & \\
\hline & $\mathrm{n}$ & $\%$ \\
\hline & 11 & 3.4 \\
\hline 44 scores & 212 & 63.1 \\
\hline $4-7$ scores & 113 & 33.5 \\
\hline
\end{tabular}

Table 9: Maternal knowledge on complementary feeding practices in Ambo town, Oromia region, Ethiopia, 2018/9.

Maternal/caregivers' knowledge score was also computed by maternal age categories, marital status, education level and occupation. Analysis of variance (ANOVA) was done to establish any significant differences in maternal 
knowledge on complementary feeding by the various maternal characteristics. There was no significant difference in the maternal knowledge score by age ( $\mathrm{p}=$ $0.097)$ and marital status $(\mathrm{p}=0.383)$ and occupation $(\mathrm{p}=$ 0.827) (Table 10). There was a significant difference in maternal knowledge scores among mothers of different education levels $(\mathrm{p}=0.017)$. Post hoc results showed that mothers who had secondary education had significantly higher total maternal knowledge score $(\mathrm{p}=0.037)$ compared to mothers who had primary education primary $(\mathrm{p}=0.017)($ Table 10$)$.

\begin{tabular}{|c|c|c|c|}
\hline \multirow[t]{2}{*}{ Maternal/caregivers' knowledge verses maternal characteristics } & & & ANOVA \\
\hline & Mean & SD & Overall and post hoc $p$ value \\
\hline Matemal age category $\mathrm{N}=336$ & 6.8 & 1.7 & 0.097 \\
\hline$<25$ years $\mathrm{N}=189$ & 6.6 & 1.7 & \\
\hline $25-34$ years $\mathrm{N}=119$ & 7.0 & 1.7 & \\
\hline 35 years and above $\mathrm{N}=28$ & 7.0 & 1.4 & \\
\hline Marital status $\mathrm{N}=336$ & 6.8 & 1.7 & 0.383 \\
\hline Single $N=54$ & 6.7 & 1.8 & \\
\hline Married N $=250$ & 6.7 & 1.7 & \\
\hline Separated $\mathrm{N}=10$ & 7.2 & 1.0 & \\
\hline Widowed $\mathrm{N}=22$ & 7.2 & 1.4 & \\
\hline Occupation $\mathrm{N}=336$ & 6.8 & 1.7 & 0.827 \\
\hline Not employed house wife $\mathrm{N}=239$ & 6.7 & 1.7 & \\
\hline Employed (salaried) $\mathrm{N}=29$ & 6.8 & 1.6 & \\
\hline Small scale trading $N=40$ & 7.0 & 1.8 & \\
\hline Casual labour $\mathrm{N}=28$ & 6.6 & 1.4 & \\
\hline Education level $\mathrm{N}=336$ & 6.8 & 1.7 & $0.017^{*}$ \\
\hline No formal education $\mathrm{N}=19$ & $7.5^{1}$ & 1.3 & 0.200 \\
\hline Primary N $=200$ & $6.5_{n}^{12}$ & 1.7 & $0.017^{* *}$ \\
\hline Secondary N = 99 & $7.0_{n}^{m}$ & 1.6 & $0.037 * 8$ \\
\hline Tertiary $\mathrm{N}=18$ & $7.2^{1}$ & 1.2 & 0.552 \\
\hline
\end{tabular}

Table 10: Maternal knowledge on infant and young child feeding by maternal characteristics in Ambo town, Oromia region, Ethiopia, 2018/9. Note: ${ }^{* *} \mathrm{P}$ values after post hoc test. Means followed by one superscript (a) are not significantly different at $\mathrm{p}=<0.05$ after post hoc while means followed by two different superscripts (ab) are significantly different.
Maternal perceptions and beliefs on complementary feeding

One FGD was held in each of the six villages at the end of quantitative data collection making a total of 6 FGDs. Each FGD had a minimum of 6 and a maximum of 12 mothers of children below 2 years of age. The discussions were guided by the questions in the focus group discussion guide.

\section{Sources and relevance of information on complementary}

\section{feeding}

Most of the mothers said that they got information regarding complementary feeding from the health facility. Mothers also reported they got information from family, friends and at the kebele 01 meeting.

The following statement made by mothers in town shows how mothers obtain information on child feeding other sources of information apart from the health facility:

"When we take our children to the health facility, health workers talk to us on how we can appropriately feed our children but we also discuss a lot on child feeding with our friends and relatives and once in a while we are sensitized by HEWs during meetings at the kebele."

Information obtained by the mothers especially from the health facility focused on dietary diversity, continued breastfeeding to 2 years and beyond, exclusive breastfeeding and the type of foods suitable for initiation of complementary feeding with soft foods. They also reported that the information they received especially from the health facilities was beneficial in ensuring that the child becomes healthy but few mothers put the information into practice due to poverty and lack of food.

The following statement demonstrates these sentiments:

"We are told by the health workers to feed our children on a balanced diet ensuring that we include foods like meat and fruits in the child's diet. We agree with the information given and we are willing to put into practice what we are 
taught but cannot afford even flour for preparing porridge!"”

\section{Introduction of complementary food, dietary diversity and frequency of complementary foods}

Mothers reported that they did not follow health worker's advice on the age of introducing complementary foods. They introduced food as early as when the infants were 2 months old because the mothers were not at home most of the times to practice exclusive breastfeeding. The following statement made by mothers in kebele 03 illustrates that mothers introduced complementary food earlier:

“We don't follow health worker's advice on introducing complementary foods. We initiate complementary foods as early as 2 months because our babies cry frequently due to hunger and we have to leave children at home and go to work to look for income"

Mothers reported that beans, mangoes, oranges, vegetable soups, mixed flour porridge, milk, avocado, tea and rice were foods commonly given to children 6-23 months old. Street foods; cooked rice and beans were also given to children. Mothers reported that meal frequency was mostly dependent on whether they had access to food or not (Table $10)$

\section{Challenges experienced on complementary feeding} practices in the study area

Mothers pointed out a number of challenges they experienced in ensuring optimal complementary feeding of their children. These included; food shortages due to inadequate income to purchase enough food, high food prices, poverty, occupations that keep mothers away from home most of the time, many children to cater for and lack of reliable jobs that can provide steady income.

\section{Factors associated with complementary feeding practices}

Socio-demographic and economic factors: age, parity, household size, sex of the household head, occupation, main source of family income, provider of food in the household. In addition, the estimated percentage of household income allocated to food and how food is obtained in the household and their association with complementary feeding practices (consumption of vitamin A rich foods, consumption of iron rich foods, minimum dietary diversity, minimum meal frequency and minimum acceptable diet) was investigated. Chi-square test was used to determine the association between categorical/nominal independent variables and the indicators of complementary feeding practices, which were also of categorical nature.

\section{Socio-demographic and economic factors and their} relationship with complementary feeding practices

Maternal age and occupation, proportion of income allocated to food and the decision maker on how family income is used were the only socio-demographic and economic factors having an association with complementary feeding practices.

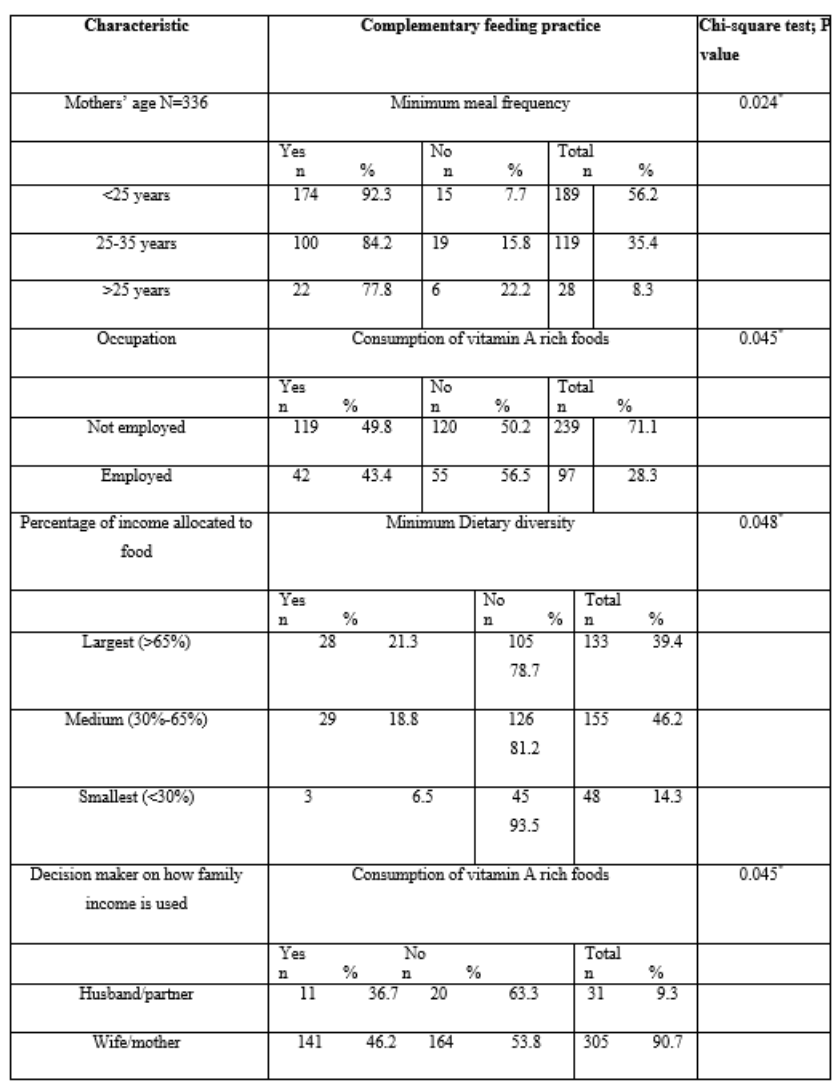

Table 11: Significant relationship between demographic and socio-economic factors and complementary feeding practices in Ambo town, Oromia region, Ethiopia, 2018/9. 
Children belonging to young mothers were more likely (chi square test; $\mathrm{p}=0.024)$ to achieve minimum meal frequency. Mothers who were not employed were more likely (chi square test; $p=0.045$ ) to feed their children with vitamin A rich foods (Table 11).

Children belonging to households which allocated more than $65 \%$ of their income to food were more (chi square test; $\mathrm{p}=0.048$ ) likely to consume a diverse diet compared to children whose households allocated less than $65 \%$ of their income to food. Children belonging to households where mothers decided on how family income is used were more likely (chi square test; $\mathrm{p}=0.045$ ) to achieve minimum dietary diversity compared to children belonging to households where husbands decided (36.7\%) on how income is used (Table 11).

The following variables; sex of the household head, mothers' age, mothers' occupation, marital status and main source of family income had no significant associations with complementary feeding practices (Table 12).

\begin{tabular}{|l|l|r|}
\hline Characterstic & Complementary feeding practice & Chi square test; P value \\
\hline Sex of the household head & Consumption of Iron rich foods & 0.433 \\
\hline Sex of the household head & Minimum meal frequency & 0.586 \\
\hline Sex of the household head & Minimum dietary diversity & 0.691 \\
\hline Sex of the household head & Minimum acceptable diet & 0.902 \\
\hline Mothers age & Consumption of Iron rich foods & 0.75 \\
\hline Mothers age & Minimum dietary diversity & 0.372 \\
\hline Mothers age & Minimum acceptable diet & 0.154 \\
\hline Mothers occupation & Consumption of Iron rich foods & 0.331 \\
\hline Mothers occupation & Minimum meal frequency & 0.262 \\
\hline Mothers occupation & Minimum dietary diversity & 0.229 \\
\hline Mothers occupation & Minimum acceptable diet & 0.126 \\
\hline Marital status & Consumption of Iron rich foods & 0.564 \\
\hline Marital status & Minimum meal frequency & 0.786 \\
\hline Marital status & Minimum dietary diversity & 0.505 \\
\hline Marital status & Minimum acceptable diet & 0.485 \\
\hline Main source of family income & Consumption of Iron rich foods & 0.535 \\
\hline Main source of family income & Minimum meal frequency & 0.873 \\
\hline Main source of family income & Minimum dietary diversity & 0.646 \\
\hline Main source of family income & Minimum acceptable diet & 0.939 \\
\hline
\end{tabular}

Table 12: Insignificant association between demographic and socio-economic factors and complementary feeding practices.

\section{Maternal/caregivers' knowledge and its relationship with} complementary feeding practices

Children belonging to mothers who knew the importance of feeding their children on a diverse diet were likely (chi square test; $\mathrm{p}=0.001)$ to achieve minimum dietary diversity compared to children belonging to mothers who were not knowledgeable. Children of mothers who knew the importance of enriching complementary foods we more likely (chi square test; $\mathrm{p}=0.007$ ) to achieve minimum acceptable diet compared to mothers who were not knowledgeable on the importance of enriching complementary foods (Table 13).

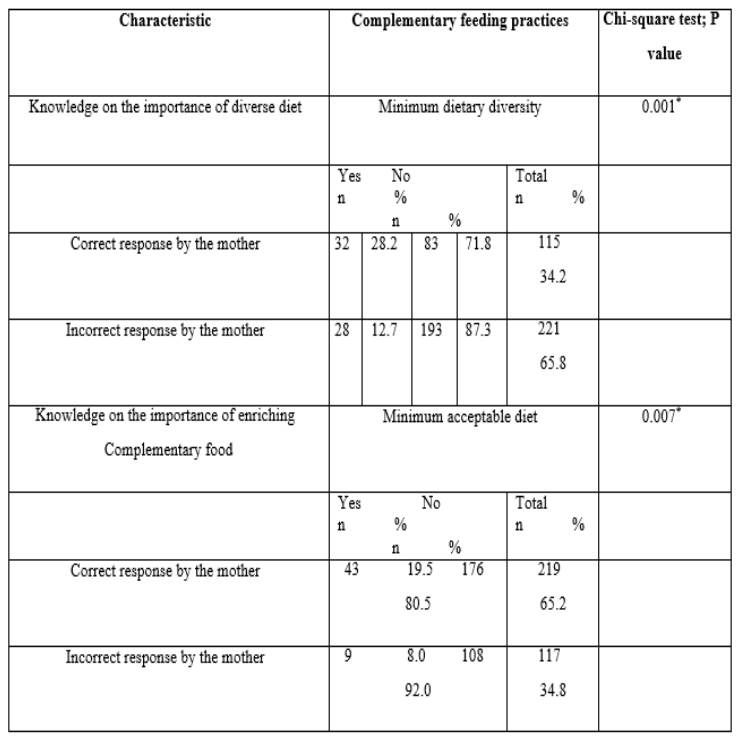

Table 13: Relationship between maternal nutrition knowledge and complementary feeding practices in Ambo town, Oromia, region, Ethiopia, 2018/9.

\begin{tabular}{|l|l|r|}
\hline Characteristic & Complementary feeding practice & Chi square test; P value \\
\hline Maternal knowledge on importance of a diverse diet & Consumption of Iron rich foods & 0.3 \\
\hline Maternal knowledge on importance of a diverse diet & Consumption of vitamin A rich foods & 0.658 \\
\hline Maternal knowledge on frequency of feeding & Minimum meal frequency & 0.788 \\
\hline $\begin{array}{l}\text { Knowledge on the importance of enriching of } \\
\text { complementary food }\end{array}$ & Consumption of vitamin A rich foods & 0.286 \\
\hline Maternal knowledge on consumption of flesh foods & Consumption of flesh foods & 0.808 \\
\hline Maternal knowledge on consumption of flesh foods & Minimum dietary diversity & 0.356 \\
\hline
\end{tabular}

Table 14: Insignificant relationship between maternal nutrition knowledge and complementary feeding practices.

Maternal knowledge on the importance of a diverse diet, frequency of feeding and importance of flesh foods had no significant association with complementary feeding practices at $\mathrm{p}>0.05$ (Table 14). 


\section{DISCUSSION}

To improve complementary feeding in low-resource settings during this critical period of growth and development, factors associated with complementary feeding practices should be investigated to provide information necessary for focused and appropriate interventions. The study adopted a cross-sectional analytical design to investigate complementary feeding practices of children aged 6 months - 23 months in Ambo town.

Socio-demographic and economic characteristics of mothers/caregivers of children 6 months - 23 months

As a whole, the participants were young, married and of primary level of education. The findings on marital status are in agreement with those conducted in other in informal settlements in Kenya and in Ethiopia by Gebru et al. [18]. The high poverty levels in Ambo town as reported by mothers during FGDs may have resulted to early school dropout by most of the girls and subsequently leading to early marriages. High levels of poverty may also lead to students discontinuing their studies because of lack of money to finance their education.

On the whole, most of the husbands were casual labourers while most of the mothers were unemployed and depended on their spouses for provision of food and other necessities. The majority of the households allocated over half of their income to food expenditure indicating high levels of poverty in the study area. As expected in an urban setting, nearly all the households obtained food through purchase. High levels of poverty, low purchasing power and lack of own production of food may have had a negative effect on the attainment of minimum acceptable diet by children aged 6 months - 23 months old in majority of the households.

Feeding practices among children 6 months - 23 months old in Ambo town

Breastfeeding practices
The minority of the mothers who did not initiate breastfeeding in this study reported that the main reason for not doing so was lack of breast milk. These findings agree with those of studies in other informal settings in Nairobi. A high percentage of the children who are one year old were still being breastfed. At 2 years of age, the rate of breastfeeding dropped to about two-thirds, indicating that one-third of the children had prematurely stopped breastfeeding and therefore missing on the benefits of the practice. A similar trend was noted in all the studies conducted by Sawadogo et al. [22] with the rate of breastfeeding decreasing from $100 \%$ at 6 months to $61 \%$ at 24 months. The median age of cessation of breastfeeding in this study was 9.0 months, a much lower rate. The relatively low duration of breastfeeding in this study could be partly explained by the fact that most mothers had to leave their children at home to look for casual jobs and thus could not continue with breastfeeding. This finding implies that a significant proportion of children were therefore likely to miss the health benefits of continued breastfeeding for the recommended duration of 2 years or more [13].

\section{Complementary feeding practices}

All the children (aged 6 months - 8 months old) in this study had appropriately been introduced to complementary feeding. Nonetheless, some of the children had been introduced to complementary foods as early as 2 months as reported by mothers during the FGDs. The finding on early introduction of complementary feeding is in agreement with those of other studies conducted in informal settlements in Kenya. Nearly all the children aged 6 months - 23 months old consumed foods made from grains, roots and tubers mainly in form of porridge. The consumption of vitamin-A rich fruits and vegetables and other fruits and vegetables was low. These findings compare with those of studies conducted in Kenya and in Nepal by Joshi et al. [23]. The low consumption of vitamin A-rich foods may have been contributed by the high poverty level in the town and therefore limited income to purchase foods. Again, the low consumption of animal origin foods may be 
contributed to the high poverty level in the town and therefore limited income to purchase foods.

The findings of this study showed that complementary feeding was low in dietary diversity. The mean dietary diversity was $(2.4 \pm 1.3)$, implying that many children ate foods from only 2 out of the 7 recommended groups [12] with the number of food groups consumed increasing with the age of the child. These findings are in agreement with those from various studies; Sawadogo et al. [22] in Burkina Faso and Joshi et al. [23] in Nepal. In a study conducted in Ethiopian [18], the minimum dietary diversity rate, was higher $(7 \%)$ than in the present study but lower than what was found in Zambia (37\%) by Disha et al. [24]. The study established that most children aged 6 months - 23 months old received one to three meals a day with a mean meal frequency of $3.5( \pm 1.1)$. In conclusion, complementary feeding, in this study was sufficient in terms of meal frequency.

\section{Mothers/caregivers knowledge and perceptions on complementary feeding in the town}

Overall, mothers' and caregivers' demonstrated a high knowledge on breastfeeding compared to complementary feeding practices. The aspects on which mothers demonstrated high knowledge included: the importance of breastfeeding and in particular the correct duration of exclusive breastfeeding; age of introduction of complementary foods and correct meal frequency, findings that are comparable to those of a study conducted in a Nairobi slum. During the FGDs mothers reported to have received knowledge on infant feeding mainly from the health facilities and this partly explains the high level of maternal knowledge on the above mentioned factors. Over two-thirds of the mothers in this study were aware of the ability of breast milk alone to sustain the baby for the first 6 months of life. Such findings are in contrast with the findings of a study conducted in Zambia where more than a third of mothers were doubtful of the nutritional adequacy of breast milk to meet the nutritional needs of an infant.
The mean maternal knowledge score on complementary feeding was $6.5 \pm 1.6$ out of a possible total score of 12 . These findings were slightly lower compared to those of in Kibera slums. The average maternal knowledge, poverty and being away from home may have been some of the factors contributing to the relatively low adherence to appropriate complementary feeding practices as reported in the FGDs.

\section{Factors associated with complementary feeding practices}

Socio-demographic and economic factors and complementary feeding practices

Several studies have established different maternal factors related to complementary feeding practices. In the present study, younger mothers and those who were not employed were significantly more likely to feed their children at the required minimum meal frequency and also to feed them on vitamin A rich foods respectively. The null hypothesis that there is no significant association between maternal demographic and socioeconomic characteristics and complementary feeding practices among children aged 6 months - 23 months in town is thus rejected. Younger mothers of whom majority were unemployed had fewer children to provide for and thus lesser workload compared to older women. This contributed to younger mothers offering better quality care to their children.

More children belonging to households where mothers decided on how family income is used significantly achieved minimum dietary diversity compared to those in households where husbands decided on how family income was used. This is because mothers are likely to purchase food items in the household when they are the ones deciding on how family income will be used compared to when fathers are the ones doing it. The proportion of total income allocated to food was also related to complementary feeding practices. Significantly, more children from households which allocated more than twothirds of their income to food consumed a diverse diet compared to those from households in which less than two- 
http://www.tridhascholars.org | June-2020

thirds of the income was allocated to food. The more income a household spends on purchasing food, the more is the likelihood that the children will achieve the minimum dietary diversity.

\section{Maternal knowledge and complementary feeding practices}

Maternal nutritional knowledge about appropriate food and feeding practices significantly influences complementary feeding practices and is often a greater determinant of malnutrition than the lack of food [3]. Mothers should therefore be equipped with the necessary knowledge on complementary feeding practices.

Mothers who were aware of the importance of a diverse diet were more likely to feed their children on a diverse diet. On the other hand, mothers who were aware of the importance of enriching complementary foods fed their children with a minimum acceptable diet. The findings emphasize the importance of maternal nutrition knowledge as a tool towards achieving appropriate complementary feeding in children aged 6 months - 23 months old. The null hypothesis that there is no significant association between maternal/caregivers' knowledge on complementary feeding practices and complementary feeding practices among children aged 6 months - 23 months in town was thus rejected. The findings on the positive association between maternal knowledge and complementary feeding practices agree with those conducted in northern Ethiopia [9], which showed that mothers with greater knowledge of healthy eating habits choose to give nutritious foods to their children.

\section{REFERENCES}

1. WHO (2016b) Indicators for assessing infant and young child feeding practices: Part 3. Country profiles, Washington DC, USA.

2. Hussein AK (2005) Breastfeeding and complementary feeding practices in Tanzania. East African Journal of Public Health 2(1): 27-31.

3. Aggarwal A, Verma S, Faridi MMA (2008) Complementary feeding-reasons for inappropriateness in timing, quantity and consistency. The Indian Journal of Pediatrics 75(1): 49-53.

4. WHO (2016a) Indicators for assessing infant and young child feeding practices: Part 2: Measurement. Conclusions of a consensus meeting held in Washington DC, USA.

5. Saha KK, Frongillo EA, Alam DS, et al. (2008) Appropriate infant feeding practices result in better growth of infants and young children in rural Bangladesh. The American Journal of Clinical Nutrition 87(6): 1852-1859.

6. Arimond M, Daelmans B, Dewey K (2008) Indicators for feeding practices in children. Lancet (London, England), 371(9612): 541-542.

7. WHO (2013a) Indicators for assessing infant and young child feeding practices: Part 1. Conclusions of a consensus meeting held in Washington DC, USA.

8. UNICEF (2013b) Tracking progress in child and maternal nutrition. A survival and development priority. United Nations children's fund. New York, USA.

9. Belete Y, Awraris W, Muleta M (2017) Appropriate complementary feeding practice was relatively low and associated with mother's education, family income, and mother's age: A community based cross-sectional study in Northern Ethiopia, Debre Markos University, Ethiopia. Journal of Nutritional Health and Food Engineering 6(2): 34-46.

10. Ethiopia demographic and health survey (2016) Central Statistical Agency; Addis Ababa, Ethiopia.

11. Black RE, Allen LH, Bhutta ZA, et al. (2008) Maternal and child undernutrition: Global and regional exposures and health consequences. The Lancet 371(9608): 243-260. 
http://www.tridhascholars.org | June-2020

12. WHO (2008) Strengthening action to improve feeding of infants and young children 6-23 months of age in nutrition and child health programmes: Report of proceedings; Geneva.

13. UNICEF (2011) The state of the world's children report. United Nations Children Fund. New York, USA.

14. UNICEF (2013a) The state of the world's children report. United Nations Children Fund. New York, USA.

15. Jones G, Steketee RW, Black RE, et al. (2003) How many child deaths can we prevent this year?. The Lancet 362(9377): 65-71.

16. Ramji S (2009) Impact of infant \& young child feeding \& caring practices on nutritional status \& health. Indian Journal of Medical Resources 130(5): 624-626.

17. Nti CA, Lartey A (2007) Young child feeding practices and child nutritional status in rural Ghana. International Journal of Consumer Studies 31(4): 326-332.

18. Gebru S (2007) Assessment of breastfeeding practice in Yeka sub-city Addis Ababa, Ethiopia. MSc thesis. Addis Ababa University, School of Graduate Studies.

19. Kassa T, Meshesha B, Haji Y, et al. (2016) Appropriate complementary feeding practices and associated factors among mothers of children age 6-23 months in Southern Ethiopia, 2015. BMC Pediatrics 16(1): 131.

20. WHO (2013) Guideline: Updates on the management of severe acute malnutrition in infants and children. Geneva.

21.fantaproject.org/sites/default/files/resources/IYCF_Datasets_Summary_2006.pdf

22. Sawadogo SP, Yves MP, Claire MR, et al. (2010) Late introduction and poor diversity were the main weaknesses of complementary foods in a cohort study in rural Burkina Faso. Nutrition 26(7-8): 746-752.

23. Joshi N, Agho KE, Dibley MJ, et al. (2012) Determinants of inappropriate complementary feeding practices in young children in Nepal: Secondary data analysis of demographic and health survey 2006. Maternal \& Child Nutrition 8: 45-59.

24. Disha AD, Rawat R, Subandoro A, et al. (2012) Infant and young child feeding (IYCF) practices in Ethiopia and Zambia and their association with child nutrition: Analysis of demographic and health survey data. African Journal of Food, Agriculture, Nutrition and Development 12(2): 5895-5914. 